\title{
Corrigenda to the Approved Lists of Bacterial Names Edited for the International Committee on Systematic Bacteriology by
}

\author{
L. R. HILL, ${ }^{1}$ V. B. D. SKERMAN, ${ }^{2}$ AND P. H. A. SNEATH ${ }^{3 *}$ \\ National Collection of Type Cultures, Central Public Health Laboratory, London, NW9, England ${ }^{1}$; Department of \\ Microbiology, University of Queensland, St. Lucia, Queensland 4067, Australia ${ }^{2}$; and Department of Microbiology, \\ Leicester University, Leicester, LEI 7RH, England ${ }^{3}$
}

\begin{abstract}
This list contains factual corrections to the Approved Lists of Bacterial Names (2) that have been brought to the attention of the International Committee on Systematic Bacteriology. Some of the matters referred to the editors concern taxonomic problems, and enquirers have been advised in such cases to consider referring the matter to the Judicial Commission. The Approved Lists is the new starting document for bacterial names, and it is therefore noted that cases of omission from the Approved Lists of well-established names should be remedied by reviving the names under Rule 28a of the International Code of Nomenclature of Bacteria (1).
\end{abstract}

Therefore, the present list contains factual corrections on matters such as bibliographic and typographic errors and orthography. A small number of author citations have been amended where it is clear that this should be done. In a work as complex as the Approved Lists, it has not been possible to check all details against the earlier literature, so it is likely that a few errors remain undetected. However, it is hoped that this list contains the great majority of errors.

\begin{tabular}{|c|c|c|c|c|c|}
\hline \multirow{2}{*}{ Page(s) } & \multirow{2}{*}{ Column } & \multirow{2}{*}{ Paragraph(s) } & \multirow{2}{*}{ Line(s) } & \multicolumn{2}{|c|}{ Change } \\
\hline & & & & From & To \\
\hline 227 & 1 & 9 & 2 & Gottingen, & Schnittspahnstrasse, Darmstadt, \\
\hline 236 & 1 & 2 & 5 & See below & $\begin{array}{l}\text { Enterobacteriaceae Rahn } 1937 \\
\text { Rahn, O. 1937. Zentralblatt fur } \\
\text { Bakteriologie, Parasitenkunde und } \\
\text { Hygiene. Abteilung II. 94:369-403. Type } \\
\text { genus Escherichia Castellani and } \\
\text { Chalmers } 1919\end{array}$ \\
\hline 236 & 1 & 9 & $1-6$ & † Editor's note . . . 1979). & Omit \\
\hline 236 & 2 & 1 & 1 & Hovind-Houger 1978 & Hovind-Hougen 1979 \\
\hline 238 & 1 & 1 and 2 & & & These paragraphs should be reversed \\
\hline 242 & 2 & 1 & 7 & $9: 771-775$ & $19: 771-775$ \\
\hline 244 & 1 & 7 & 1 & Palleroni 1978 & Palleroni 1979 \\
\hline 244 & 1 & 7 & 2 & Palleroni, N.J. 1978. & Palleroni, N.J. 1979. \\
\hline 244 & 1 & 7 & 5 & Palleroni, N.J. 1978. & Palleroni, N.J. 1979. \\
\hline 247 & 2 & 4 & 1 & pacifus & pacificus \\
\hline 250 & 2 & 3 & 6 & Type species: $A$. serpens & Type species: $A$. serpens (Müller 1786 ) \\
\hline 252 & 2 & 3 & 1 & Muller & Müller \\
\hline 252 & 2 & 3 & 3 & Muller & Müller \\
\hline 254 & 1 & 5 & 1 & Dubinin & Dubinina \\
\hline 254 & 1 & 5 & 2 & Dubinin & Dubinina \\
\hline 255 & 1 & 4 & 1 & A. brasiliense & A. brasilense \\
\hline 263 & 2 & 4 & $1-4$ & (Fujino, Miwatani, . . . Tamura 1965) & $\begin{array}{l}\text { (Fujino, Okuno, Nakada, Aoyama, Fukai, } \\
\text { Mukai and Ueho 1951) }\end{array}$ \\
\hline 263 & 2 & 4 & $6-10$ & Fujino, T., T. Miwatani, . . . 8:63-71; & $\begin{array}{l}\text { Fujino, T., Y. Okuno, D. Nakada, A. } \\
\text { Aoyama, K. Fukai, T. Mukai and T. } \\
\text { Ueho. 1951. Journal of the Japanese } \\
\text { Association of Infectious Diseases 25:11; }\end{array}$ \\
\hline 265 & 2 & 5 & 1 & thermophilium & thermophilum \\
\hline 282 & 2 & 4 & 5 & $10: 51-545$ & $10: 517-545$ \\
\hline
\end{tabular}

* Corresponding author. 


\begin{tabular}{|c|c|c|c|c|c|}
\hline \multirow{2}{*}{ Page(s) } & \multirow{2}{*}{ Column } & \multirow{2}{*}{ Paragraph(s) } & \multirow{2}{*}{ Line(s) } & \multicolumn{2}{|c|}{ Change } \\
\hline & & & & From & To \\
\hline 284 & 2 & 2 & $1-2$ & Fontaine, Peterson, McCoy and Johnison & $\begin{array}{l}\text { Fontaine, Peterson, McCoy, Johnson and } \\
\text { Ritter }\end{array}$ \\
\hline 284 & 2 & 2 & $3-4$ & $\begin{array}{l}\text { Fontaine, F. E., W. H. Peterson, E. McCoy } \\
\text { and M. J. Johnson }\end{array}$ & $\begin{array}{l}\text { Fontaine, F. E., W. H. Peterson, E. } \\
\text { McCoy, M. J. Johnson and G. J. Ritter }\end{array}$ \\
\hline 284 & 2 & 2 & $7-8$ & $\begin{array}{l}\text { Fontaine, F. E., W. H. Peterson, E. McCoy } \\
\text { and M. J. Johnson }\end{array}$ & $\begin{array}{l}\text { Fontaine, F. E., W. H. Peterson, E. } \\
\text { McCoy, M. J. Johnson and G. J. Ritter }\end{array}$ \\
\hline 286 & 2 & 5 & $1-3$ & $\begin{array}{l}\text { (Schuster, Hoff, Mandel and Lazar 1973) } \\
\text { emend. Vidaver and Mandel } 1974\end{array}$ & Vidaver and Mandel 1974 \\
\hline 286 & 2 & 5 & $4-8$ & $\begin{array}{l}\text { Schuster, M. L., B. Hoff, M. Mandel and I. } \\
\text { Lazar. 1973. 27th Annual Corn and } \\
\text { Sorghum Research Conference } \\
\text { Proceedings, 1972, 27:176-191; Vidaver, } \\
\text { A. K. and M. Mandel. }\end{array}$ & Vidaver, A. K. and M. Mandel. \\
\hline 291 & 2 & 1 & 2 & 110:1-10. & $110: 3-12$. \\
\hline 292 & 2 & 4 & 6 & brasiliensis & brasiliense \\
\hline 292 & 2 & 5 & 1 & brasiliensis & brasiliense \\
\hline 293 & 1 & 6 & 3 & 7:1854-1855. & $7: 1853-1855$ \\
\hline 295 & 2 & 7 & 5 & $71: 1$ & $71: 1-7$ \\
\hline 300 & 1 & 5 & 1 & halmephilium & halmephilum \\
\hline 300 & 2 & 5 & 5 & 102:113. & 113:219-225. \\
\hline 304 & 2 & 1 & $1-2$ & (Asai 1935) Asai, Iizuka and Komagata 1964 & Asai 1935 \\
\hline 304 & 2 & 1 & $4-7$ & $\begin{array}{l}\text {; Asai, T., H. lizuka and K. Komagata } \\
\text { 1964. Journal of General and Applied } \\
\text { Microbiology 10:95-126. }\end{array}$ & omit \\
\hline 306 & 1 & 7 & 1 & Sniezko & Snieszko \\
\hline 306 & 1 & 7 & 2 & Sniezko & Snieszko \\
\hline 306 & 1 & 8 & $1-2$ & (Matthews and Pattison 1961) Shope 1964 & Shope 1964 \\
\hline 306 & 1 & 8 & $3-5$ & $\begin{array}{l}\text { Matthews, P. R. J. and I. H. Pattison. } 1961 . \\
\text { Journal of Comparative Pathology 71:44- } \\
52 \text {; }\end{array}$ & omit \\
\hline 308 & 2 & 4 & 1 & J. lividum De Ley, Segers and Willis 1978 & $\begin{array}{l}\text { J. lividum (Eisenberg (1891) De Ley, Segers } \\
\text { and Gillis } 1978\end{array}$ \\
\hline 308 & 2 & 4 & 2 & De Ley, J., P. Segers and M. Gillis. & $\begin{array}{l}\text { Eisenberg, J. 1891. Bacteriologische } \\
\text { Diagnostik, Hilfstabellen zum Gebrauche } \\
\text { beim Praktischen Arbeiten, 3rd ed. } \\
\text { Leopold Voss, Hamburg; De Ley, J., P. } \\
\text { Segers and M. Gillis. }\end{array}$ \\
\hline 309 & 2 & 6 and 7 & & & $\begin{array}{l}\text { The order of these paragraphs should be } \\
\text { reversed. }\end{array}$ \\
\hline 310 & 1 & 5 & 4 & multiparis & multipara \\
\hline 310 & 1 & 6 & 1 & multiparis & multipara \\
\hline 311 & 1 & 6 & 1 & catenaforme & catenaformis \\
\hline 311 & 2 & 3 & 3 & $35: 463$. & $35: 463-471$ \\
\hline 314 & 1 & 2 & 1 & Kandler and Abo-Elnaga 1966 & Niven and Evans 1957 \\
\hline 314 & 1 & 2 & $2-5$ & $\begin{array}{l}\text { Kandler, O. and I. G. Abo-Elnaga. } 1966 . \\
\text { Zentralblatt fur Bakteriologie, } \\
\text { Parasitenkunde, Infektionskrankheiten } \\
\text { und Hygiene. Abteilung II. 120:753-754. }\end{array}$ & $\begin{array}{l}\text { Niven, C. F. and J. B. Evans. 1957. Journal } \\
\text { of Bacteriology 73:758-759. }\end{array}$ \\
\hline 317 & 1 & 4 & $3-5$ & Lauterborn, R. 1915. . . 13:395-481. & $\begin{array}{l}\text { Lauterborn, R. } 1915 \ldots \text { 13:395-481; } \\
\text { Utermohl, H. and F. Koppe. } 1924 \text { in } \\
\text { Koppe, F. Archiv fur Hydrobiologie } \\
\text { 14:619-672. }\end{array}$ \\
\hline 317 & 2 & 3 & 4 & not & not cultivated \\
\hline 318 & 1 & 5 & 3 & not & not cultivated \\
\hline 318 & 2 & 2 & 9 & not & not cultivated \\
\hline 324 & 2 & 4 & $5-7$ & $\begin{array}{l}\text { Brenner, Farmer, Fanning, Steigerwalt, } \\
\text { Klykken, Wathen, Hickman and Ewing } \\
1978\end{array}$ & Fulton 1943 \\
\hline 324 & 2 & 4 & $10-12$ & $\begin{array}{l}\text { Brenner, Farmer, Fanning, Steigerwalt, } \\
\text { Klykken, Wathen, Hickman and Ewing } \\
1974\end{array}$ & Fulton 1943 \\
\hline 324 & 2 & 4 & $15-20$ & $\begin{array}{l}\text { Brenner, D. J., J. J. Farmer III, G. R., } \\
\text { Fanning, A. G. Steigerwalt, P. Klykken, } \\
\text { H. G. Wathen, F. W. Hickman and } \\
\text { W. H. Ewing. 1978. International Journal } \\
\text { of Systematic Bacteriology 28:269-282. }\end{array}$ & $\begin{array}{l}\text { Fulton, MacD. 1943. Journal of } \\
\text { Bacteriology 46:79-81. }\end{array}$ \\
\hline 324 & 2 & 4 & 25 & and W. H. Ewing. 1978. Ibid. & $\begin{array}{l}\text { and W. H. Ewing. 1978. International } \\
\text { Journal of Systematic Bacteriology } \\
28: 269-282 \text {. }\end{array}$ \\
\hline 325 & 1 & 6 & 1 & chelonei & chelonae \\
\hline 325 & 1 & 7 & 1 & chelonei & chelonae \\
\hline 325 & 2 & 2 & 1 & chelonei subsp. chelonei & chelonae subsp. chelonae \\
\hline
\end{tabular}

Continued on following page 


\begin{tabular}{|c|c|c|c|c|c|}
\hline \multirow{2}{*}{ Page(s) } & \multirow{2}{*}{ Column } & \multirow{2}{*}{ Paragraph(s) } & \multirow{2}{*}{ Line(s) } & \multicolumn{2}{|c|}{ Change } \\
\hline & & & & From & To \\
\hline 327 & 1 & 3 & 6 & p. 703 . & pp. 703-704. \\
\hline 327 & 1 & 6 & 1 & Tsukamura 1966 & Tsukamura, Toyama and Mizuno 1965 \\
\hline 327 & 1 & 6 & $2-3$ & $\begin{array}{l}\text { Tsukamura, M. 1966. Journal of General } \\
\text { Microbiology 42:7-12. }\end{array}$ & $\begin{array}{l}\text { Tsukamura, M., H. Toyama and S. Mizuno. } \\
\text { 1965. Medicine and Biology, Tokyo } \\
\text { 70:232-235. }\end{array}$ \\
\hline 334 & 1 & 7 & $2-4$ & $\begin{array}{l}\text { Zentralblatt fur Bakteriologie, } \\
\text { Parasitenkunde, Infektionskrankheiten } \\
\text { und Hygiene. Abteilung II. 147:158-161. }\end{array}$ & $\begin{array}{l}\text { Zeitschrift fur Hygiene und } \\
\text { Infektionskrankheiten 147:158-161. }\end{array}$ \\
\hline 334 & 1 & 8 & $2-4$ & $\begin{array}{l}\text { Zentralblatt fur Bakteriologie, } \\
\text { Parasitenkunde, Infektionskrankheiten } \\
\text { und Hygiene. Abteilung II. 148:445-457. }\end{array}$ & $\begin{array}{l}\text { Zeitschrift fur Hygiene und } \\
\text { Infektionskrankheiten 148:445-457. }\end{array}$ \\
\hline 334 & 2 & 2 & $2-5$ & $\begin{array}{l}\text { Zentralblatt fur Bakteriologie, } \\
\text { Parasitenkunde, Infektionskrankheiten } \\
\text { und Hygiene. Abteilung II. 148:445-457. }\end{array}$ & $\begin{array}{l}\text { Zeitschrift fur Hygiene und } \\
\text { Infektionskrankheiten 148:445-457. }\end{array}$ \\
\hline 334 & 2 & 3 & $2-4$ & $\begin{array}{l}\text { Zentralblatt fur Bakteriologie, } \\
\text { Parasitenkunde, Infektionskrankheiten } \\
\text { und Hygiene. Abteilung II. 148:445-457. }\end{array}$ & $\begin{array}{l}\text { Zeitschrift fur Hygiene und } \\
\text { Infektionskrankheiten 148:445-457. }\end{array}$ \\
\hline 335 & 1 & 5 & 1 & Tult & Thibault \\
\hline 336 & 1 & 5 & $4-5$ & General Systemic Bacteriology & General Systematic Bacteriology \\
\hline 336 & 1 & 6 & 3 & 10:274-289; & 10:R274-R289; \\
\hline 336 & 2 & 1 & 5 & $76: 83-203$ & 76:183-203. \\
\hline 338 & 1 & 4 & 1 & otitidis-caviarum & otitidiscaviarum \\
\hline 338 & 2 & 4 & 1 & Meyer 1976 & (Brocq-Rousseau 1904) Meyer 1976 \\
\hline 338 & 2 & 4 & $2-3$ & $\begin{array}{l}\text { Meyer, J. 1976. International Journal of } \\
\text { Systematic Bacteriology 26:487-493. }\end{array}$ & $\begin{array}{l}\text { Brocq-Rousseau, D. 1904. Revue Générale } \\
\text { de Botanique 16:219-230; Meyer, J. } 1976 . \\
\text { International Journal of Systematic } \\
\text { Bacteriology 26:487-493. }\end{array}$ \\
\hline 338 & 2 & 4 & 4 & Meyer 1976 & (Brocq-Rousseau 1904) Meyer 1976 \\
\hline 338 & 2 & 5 & 1 & Meyer 1976 & (Brocq-Rousseau 1904) Meyer 1976 \\
\hline 338 & 2 & 5 & $2-3$ & $\begin{array}{l}\text { Meyer, J. 1976. International Journal of } \\
\text { Systematic Bacteriology 26:487-493 }\end{array}$ & $\begin{array}{l}\text { Brocq-Rousseau, D. 1904. Revue Générale } \\
\text { de Botanique 16:219-230; Meyer, J. } 1976 . \\
\text { International Journal of Systematic } \\
\text { Bacteriology 26:487-493. }\end{array}$ \\
\hline 340 & 1 & 2 & 5 & guilliermondi & guilliermondii \\
\hline 340 & 1 & 3 & 1 & guilliermondi & guilliermondii \\
\hline 345 & 2 & 5 & 1 & Pleisomonas & Plesiomonas \\
\hline 346 & 1 & 8 & 1 & acidi-propionici & acidipropionici \\
\hline 355 & 1 & 2 & 3 & 218:661. & 218:661-674. \\
\hline 356 & 1 & 5 & 3 & Alderson 1979 & Alderson 1977 \\
\hline 356 & 1 & 5 & $9-11$ & $\begin{array}{l}\text { Goodfellow, M. and G. Alderson. } 1979 . \\
\text { International Journal of Systematic } \\
\text { Bacteriology 29:79-80; }\end{array}$ & omit \\
\hline 356 & 1 & 6 & 2 & Alderson 1979 & Alderson 1977 \\
\hline 356 & 1 & 6 & $5-7$ & $\begin{array}{l}\text { Goodfellow, M. and G. Alderson. } 1979 . \\
\text { International Journal of Systematic } \\
\text { Bacteriology 29:79-80; }\end{array}$ & omit \\
\hline 356 & 2 & 5 & 2 & 1979 & 1977 \\
\hline 356 & 2 & 5 & $5-7$ & $\begin{array}{l}\text { Goodfellow, M. and G. Alderson. } 1979 . \\
\text { International Journal of Systematic } \\
\text { Bacteriology 29:79-80; }\end{array}$ & omit \\
\hline 356 & 2 & 6 & $1-2$ & Goodfellow and Alderson 1979 & Tsukamura 1974 \\
\hline 357 & 1 & 1 & $1-6$ & $\begin{array}{l}\text { Goodfellow, M. and G. Alderson. } 1979 . \\
\text { International Journal of Systematic } \\
\text { Bacteriology 29:79-80; Effective } \\
\text { publication Goodfellow, M. and G. } \\
\text { Alderson. 1977. Journal of General } \\
\text { Microbiology 100:99-122. }\end{array}$ & $\begin{array}{l}\text { Tsukamura, M. 1974. Japanese Journal of } \\
\text { Microbiology 18:37-44. }\end{array}$ \\
\hline 357 & 1 & 7 & 1 & Kluyver and van Niel 1937 & Czurda and Maresch 1937 \\
\hline 357 & 1 & 7 & 2 & Kluyver, A. J. and C. B. van Niel & Czurda, V. and E. Maresch \\
\hline 360 & 2 & 1 & 3-4 & $88: 76-78$ & $88: 75-85$. \\
\hline 360 & 2 & 2 & $3-4$ & $88: 76-78$. & 88:75-85. \\
\hline 360 & 2 & 3 & 4 & cholerae-suis & choleraesuis \\
\hline 360 & 2 & 5 & 1 & cholerae-suis & choleraesuis \\
\hline 362 & 1 & 2 & 3 & Scienz & Scienze \\
\hline 362 & 1 & 3 & 3 & Gavin & Gavini \\
\hline 362 & 1 & 4 & 1 & (Grimes and Hennerty 1961) & (Grimes and Hennerty 1931) \\
\hline 362 & 1 & 4 & 4 & Grimes, M. and P. J. Hennerty. 1961. & Grimes, M. and P. J. Hennerty. 1931. \\
\hline 362 & 1 & 5 & 2 & Italianna & Italiana \\
\hline
\end{tabular}




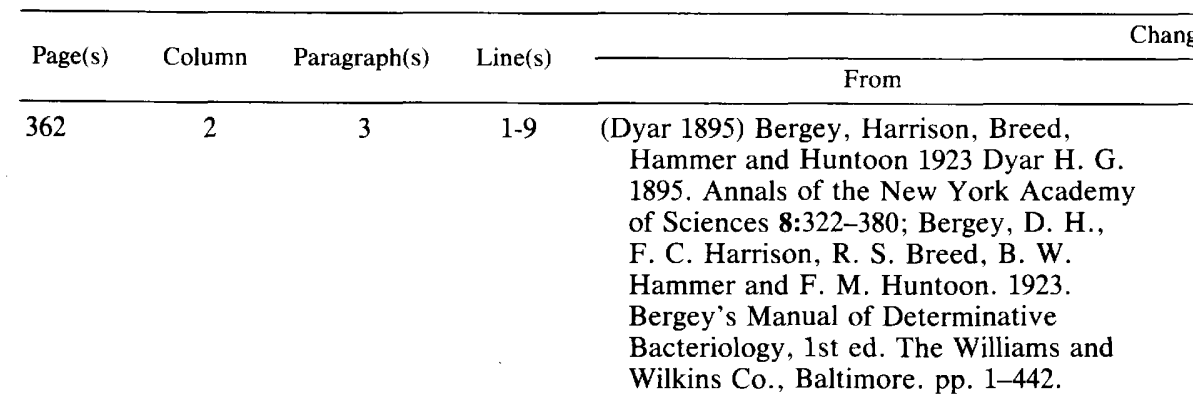

\begin{tabular}{|c|c|c|c|c|}
\hline 367 & 1 & 5 & 6 & 62:189. \\
\hline 367 & 2 & 8 & 2 & Pier, G. B. and S. H. Madin. \\
\hline 372 & 1 & 4 & 1 & Waksman 1957 \\
\hline 372 & 1 & 4 & $3-7$ & Waksman, S. A. 1957 in Breed, R \\
\hline
\end{tabular}

Waksman, S. A. 1957 in Breed, R. S.,

E. G. D. Murray and N. R. Smith (eds). Bergey's Manual of Determinative Bacteriology, 7th ed. The Williams and Wilkins Co., Baltimore. parvullus

\begin{tabular}{|c|c|c|c|c|}
\hline 395 & 2 & 5 & 1 & parvullus \\
\hline 396 & 1 & 4 & $4-5$ & Japanese Medical Journal 5:237-338. \\
\hline 400 & 2 & 3 & 1 & castelarense \\
\hline 404 & 1 & 3 & 1 & violaceoniger \\
\hline 406 & 2 & 1 & 1 & KCC A-2006 \\
\hline 406 & 2 & 3 & 1 & indianensis \\
\hline 407 & 2 & 3 & $i$ & aureoversales \\
\hline 408 & 1 & 4 & $1-2$ & Baldacci and Locci 1974 \\
\hline 408 & 1 & 4 & $3-4$ & Baldacci, E. and R. Locci. 1974. Bergey 8. \\
\hline
\end{tabular}

\begin{tabular}{|c|c|c|c|c|}
\hline 410 & 2 & 2 & 1 & olivoreticulum \\
\hline 410 & 2 & 5 & 1 & parvisporogenum \\
\hline 411 & 2 & 8 & 1 & Tsiklinsky \\
\hline 412 & 1 & 1 & 1 & Tsiklinsky, P. \\
\hline 412 & 1 & 1 & 3 & Tsiklinsky \\
\hline 412 & 1 & 3 & 1 & dichotomica \\
\hline 412 & 1 & 6 & 1 & Tsiklinsky \\
\hline 412 & 1 & 6 & 2 & Tsiklinsky, $P$. \\
\hline 415 & 2 & 4 & 6 & and Bringmann. \\
\hline 416 & 1 & 4 & 1 & paraluis-cuniculi \\
\hline 417 & 2 & 4 & $1-2$ & Sakazaki, Iwanami and Fukumi 1963 \\
\hline 417 & 2 & 4 & $4-6$ & $\begin{array}{l}\text { Sakazaki, R., S. Iwanami and H. Fukumi. } \\
\text { 1963. Japanese Journal of Medical } \\
\text { Science and Biology 16:161-188. }\end{array}$ \\
\hline
\end{tabular}

(Lehmann and Neumann 1896) Breed, Murray and Hitchens 1948

Lehmann, K. B. and R. Neumann. 1896. Atlas and Grundriss der Bakteriologie und Lehrbuch der speciellen bakteriologischen Diagnostik. 1st ed. J. F. Lehmann, Munchen; Breed, R. S., E. G. D. Murray and A. P. Hitchens. 1948. Bergey's Manual of Determinative Bacteriology, 6th ed. The Williams and Wilkins Co., Baltimore. pp. 1-1529.

62:186-189.

Pier, G. B. and S. H. Madin. 1976.

Waksman 1953

Waksman, S. A. 1953 in Waksman S. A. and $\mathrm{H}$. A. Lechevalier. Guide to the classification of the actinomycetes and their antibiotics. The Williams and Wilkins Co., Baltimore. pp. 1-246. parvulus

Japanese Journal of the Medical Sciences and Biology 5:327-339.

castelarensis

violaceusniger

KCC A-0026

indianense

aureoversile

(Benedict, Dvonch, Shotwell, Pridham and Lindenfelser 1952) Baldacci, Farina and Locci 1966

Benedict, R. G., W. Dvonch, O. L.

Shotwell, T. G. Pridham and L. A.

Lindenfelser. 1952. Antibiotics and

Chemotherapy 2:591-594; Baldacci, E.,

G. Farina and R. Locci. 1966. Giornale di Microbiologia 14:153-171.

olivoreticuli

parvisporogenes

Tsilinsky

Tsilinsky, P.

Tsilinsky

dichotomicus

Tsilinsky

Tsilinsky, P.

and G. Bringmann.

paraluiscuniculi

(Fujino, Okuno, Nakada, Aoyama, Fukai, Mukai and Ueho 1951) Sakazaki, Iwanami and Fukumi 1963

Fujino, T., Y. Okuno, D. Nakada, A.

Aoyama, K. Fukai, T. Mukai and T.

Ueho. 1951. Journal of the Japanese

Association of Infectious Diseases 25:11;

Sakazaki, R., S. Iwanami and H. Fukumi.

1963. Japanese Journal of Medical

Science and Biology 16:161-188.
The editors are grateful to the many workers who have submitted corrections and will be glad to receive any further ones. The officers of the International Committee on Systematic Bacteriology also express their thanks to all those who have contributed to the Approved Lists.

\section{LITERATURE CITED}

1. Lapage, S. P., P. H. A. Sneath, E. F. Lessel, V. B. D. Skerman, H. P. R. Seeliger, and W. A. Clark (ed.). 1975. International code of nomenclature of bacteria. 1975 Revision. American Society for Microbiology, Washington, D.C.

2. Skerman, V. B. D., V. McGowan, and P. H. A. Sneath (ed.). 1980. Approved lists of bacterial names. Int. J. Syst. Bacteriol. 30:225-420. 